\title{
A guide to the management of medical emergencies
}

By P L Ariyananda, G Hettiarachchi and T P Weerarathna. 297 pages, Upuli Offset Printers, 2010. Second Edition. ISBN 978-955-98849-5-8

A day in the life of an internist can vary from the routine dealing with "cold cases" - prescribing a few tablets and changing them if they don't work - to moments of absolute trepidation when things start to go wrong in an acutely ill patient. Making the right clinical decision within minutes could mean the difference between life and death. Any physician, senior or junior, has to be able to deal competently with these emergency situations. Though the principles of management may be known, details of what drug to use, at what dose and route may not be at one's fingertips all the time. This is why practicing doctors should have ready access to a well written emergency manual; most sensible doctors do.

The second edition of the book " $A$ guide to the management of medical emergencies" by Ariyananda and colleagues 15 years after the first edition is, therefore, welcome. It is well written and concise, and has clear instructions. When dealing with emergencies, the book, rather than just indicating names of drugs and their doses also attempts to give a brief account of the disease condition where this is relevant. The book is structured into 13 sections based on the main organ-systems involved (eg: cardiovascular, respiratory), and also has sections on shock, acute poisoning, snake bite and injuries due to physical agents. Though the coverage of cardiovascular, respiratory and neurological emergencies are exhaustive compared to some of the other organsystems, the book manages to get through almost all the important medical emergencies that are locally relevant. Most topics on which internists tend to struggle, for example metabolic and endocrine emergencies, have been dealt with lucidly.

This book is of course one among many such books and guides on management of medical emergencies. But this one is probably more useful than most to doctors practicing medicine in Sri Lanka. This is because of the excellent coverage of locally relevant topics such as snakebite, and poisoning with yellow oleander and agrochemicals. Other useful information includes lists of trade names of agro-chemicals and normal values of investigations. If I were to single out one shortcoming it is the rather brief coverage of the management of dengue shock syndrome, currently very topical. The book also does not highlight the management of complications of dengue such as liver failure and myocarditis. Lack of an evidence based consensus in the treatment of these problems may have been a reason for their omission.

Overall, this book is a "must have" for all medical students and even postgraduates practicing medicine in Sri Lanka.

A Premawardhena, Department of Medicine, Faculty of Medicine, University of Kelaniya, Ragama, Sri Lanka. e-mail<anuja@mfac.kIn.ac.lk> Competing interests: none declared. 